\title{
NUEVO REGISTRO DE CESTRUM MORTONIANUM (SOLANACEAE) Y DESCRIPCIÓN DE UNA VARIEDAD EN LA VERTIENTE PACÍFICA DE MÉXICO
}

\author{
Ramón Cuevas ${ }^{1}$ y Juan Carlos Montero ${ }^{2}$ \\ ${ }^{1}$ Universidad de Guadalajara, Instituto Manantlán de Ecología y Conservación de \\ la Biodiversidad, Centro Universitario de la Costa Sur, Avenida Independencia \\ Nacional 151, 48900 Autlán de Navarro, Jalisco, México, rcuevas@cucsur.udg.mx \\ ${ }^{2}$ Universidad Michoacana de San Nicolás de Hidalgo, Facultad de Biología, \\ Edificio "R”, Ciudad Universitaria, Avenida Fco. J. Mújica s/n., Col. Felícitas del \\ Río, 58030 Morelia, Michoacán, México
}

\section{RESUMEN}

Se revisa la distribución de Cestrum mortonianum y se describe e ilustra una variedad de la Sierra de Manantlán. Se discuten las características que la diferencian de $C$. mortonianum var. mortonianum, C. tomentosum y C. pacificum, las cuales tienen en común la presencia de pubescencia ramificada. Se proporciona una clave para separar las especies de Cestrum de México con este tipo de indumento.

Palabras clave: Cestrum, México, Sierra de Manantlán, Solanaceae.

\section{ABSTRACT}

We present a review of the geographic distribution of Cestrum mortonianum, as well as a description and an illustration of a new variety from the Sierra de Manantlan. The features that differentiate this variety from $C$. mortonianum var. mortonianum, and from $C$. tomentosum and C. pacificum -all of which share the presence of branched pubescence - are also discussed. A key to distinguish the Mexican species of Cestrum whith this indumenta is provided.

Key words: Cestrum, México, Sierra de Manantlán, Solanaceae. 
Cestrum es un género de origen americano, con algunos taxones naturalizados en otros continentes (Francey, 1935; Nee, 2001; Nee, en preparación). Su número de especies aún no ha sido bien definido, pero se considera que debe estar entre 150 y 175 (D’ Arcy, 2001; Nee, 2001). Se trata de un género complicado, dentro del cual aún existen complejos taxonómicos que deben ser resueltos; tal es el caso de los miembros con indumento de pelos ramificados, relacionados con C. tometosum L. f., una especie que se conoce desde el norte de México hasta Perú.

Durante la preparación de la Flora de Manantlán, el Dr. Michael Nee hizo la anotación a ejemplares procedentes de la Sierra de Manantlán, como cercanos a C. tomentosum, pero con algunas características diferentes que lo llevaron a considerar que podría tratarse de una entidad nueva y así fue consignada en la Flora de Manantlán (Vázquez et al., 1995).

Después de hacer revisiones críticas del material de Cestrum con pubescencia ramificada de la Sierra de Manantlán y de otras partes de la vertiente pacífica de México, concluimos que además de $C$. tomentosum existe otro taxon relacionado, el cual coincide con la mayoría de las características registradas para C. mortonianum J. L. Gentry, una especie distribuida en Guatemala (Gentry y Standley, 1974). En Cestrum, la indumentación, la longitud del cáliz y la corola y la presencia o ausencia de apéndice en los filamentos y su posición de inserción en el tubo de la corola han sido caracteres fundamentales para separar taxones (Francey, 1935; Nee, 1986); el hecho de que los ejemplares de la Sierra de Manantlán en forma consistente carezcan de indumento en la parte externa del tubo de la corola, así como su distribución disyunta justifican la propuesta de una nueva variedad.

Cestrum mortonianum J. L. Gentry var. jardelii Cuevas \& Montero. Figs. 1 y 2.

Arbor 5-10 m alta; truncus usque $35 \mathrm{~cm}$ diametro; indumentum dendroideum; folia ovato-lanceolata vel late elliptica, membranacea, 11-21.5 cm longa, 5.5-10 cm lata, supra puberula vel glabrata, subtus puberula vel tomentosa; petioli 2-4.3 cm longi; inflorescentiae $2.5-10.5 \mathrm{~cm}$ longae, bracteolae lineares; calyx 4-6 mm longus, tomentosus; corolla viridi-luteola, $1.5-2.3 \mathrm{~cm}$ longa, extus glabra; filamenta ad 3/4 longitudinem tubo adnata, dentata et pilosa, antherae 1-1.6 mm longae; ovarium 1.2$1.7 \mathrm{~mm}$ crassum; bacca 7-8 mm longa, glabra; semina 2.8-4.5 mm longa.

Árbol perennifolio, 5-10 m de altura, hasta de 35 cm de diámetro; corteza externa grisácea, la interna verde-amarillenta; indumento de tricomas dendroides; ramillas de 2-4 mm de diámetro, glabrescentes a tomentosas; hojas alternas, la lámi- 


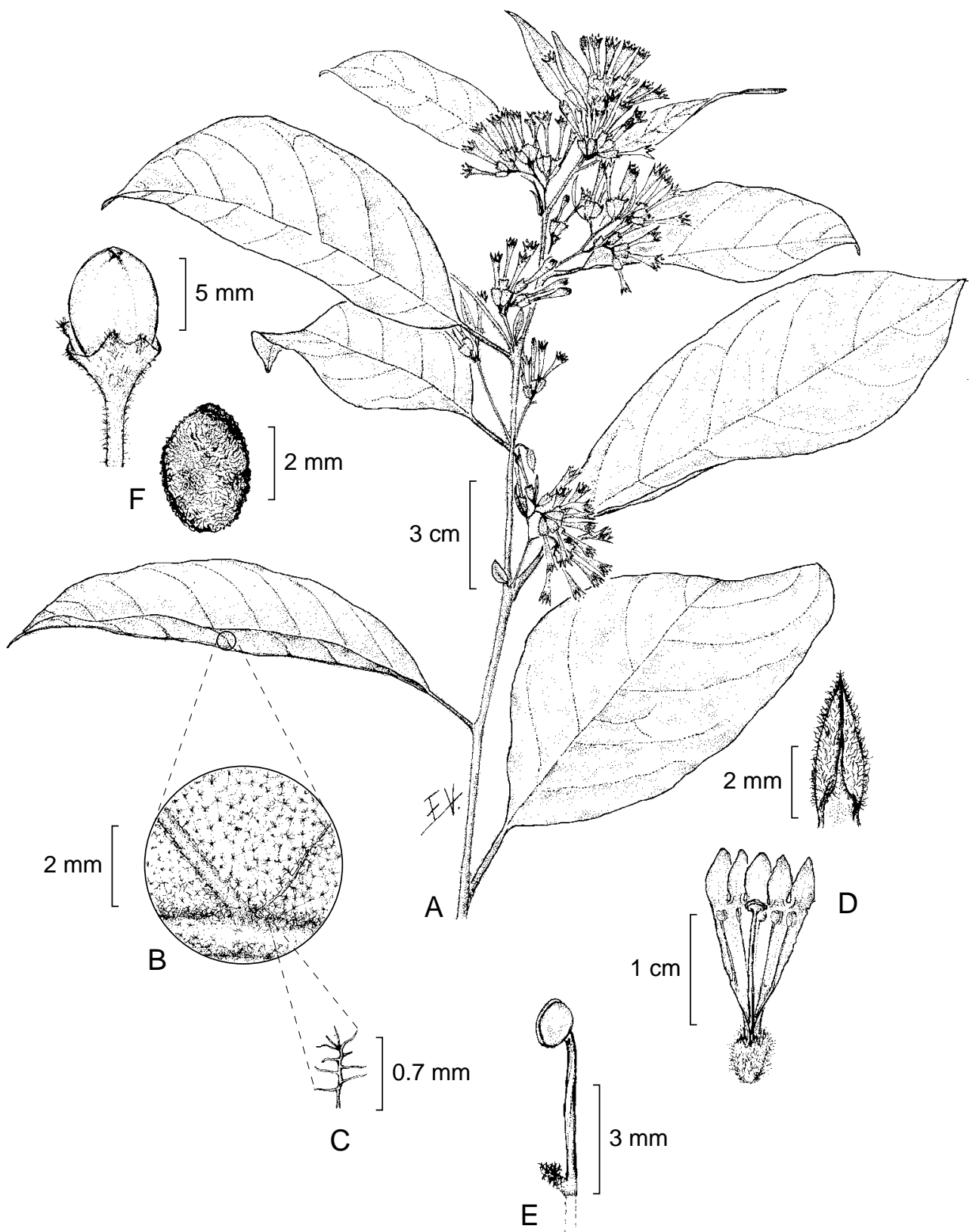

Fig. 1. Cestrum mortonianum var. jardelii. A. ramilla con flores; B. indumento de la hoja por el envés; C. tricoma ramificado; D. flor abierta y detalle de un lóbulo de la corola por su parte interna E. parte de un estambre mostrando la antera y el apéndice; F. fruto y semilla (Cuevas y Núñez 8902). 
na ovado-lanceolada a anchamente elíptica, en ocasiones oblonga, membranosa, 11$21.5 \mathrm{~cm}$ de largo, 5.5-10 cm de ancho, el ápice acuminado, algunas veces agudo, rara vez redondeado, el margen entero, en ocasiones levemente sinuado, la base cuneada, algunas veces redondeada a asimétrica, con 10-12 pares de nervios secundarios, el haz levemente áspero, pubérulo a glabrescente, los nervios hundidos, el envés pubérulo a tomentoso, los nervios prominentes; pecíolo 2-4.3 cm de largo, acanalado en la parte adaxial, pubérulo a tomentoso, verde-amarillento en seco; inflorescencias racemosas a paniculadas, axilares a subterminales, 2.5-10.5 cm de largo, con 5-50 flores; pedúnculos hasta de $5 \mathrm{~mm}$ de largo; bractéolas lineares y caducas; cáliz 4-6 mm de largo, tomentoso, los pedicelos hasta de $2 \mathrm{~mm}$ de largo, los lóbulos 5, subiguales, ovados, 0.5-1.5 mm de largo, con el ápice agudo a obtuso; corola tubular, ampliándose gradualmente hacia el ápice, 1.5-2.3 cm de larga, verde-amarillenta, el exterior glabro, con excepción de los márgenes y ápices de los lóbulos, éstos tomentosos a glabrescentes externamente y tomentosos en su interior, los lóbulos

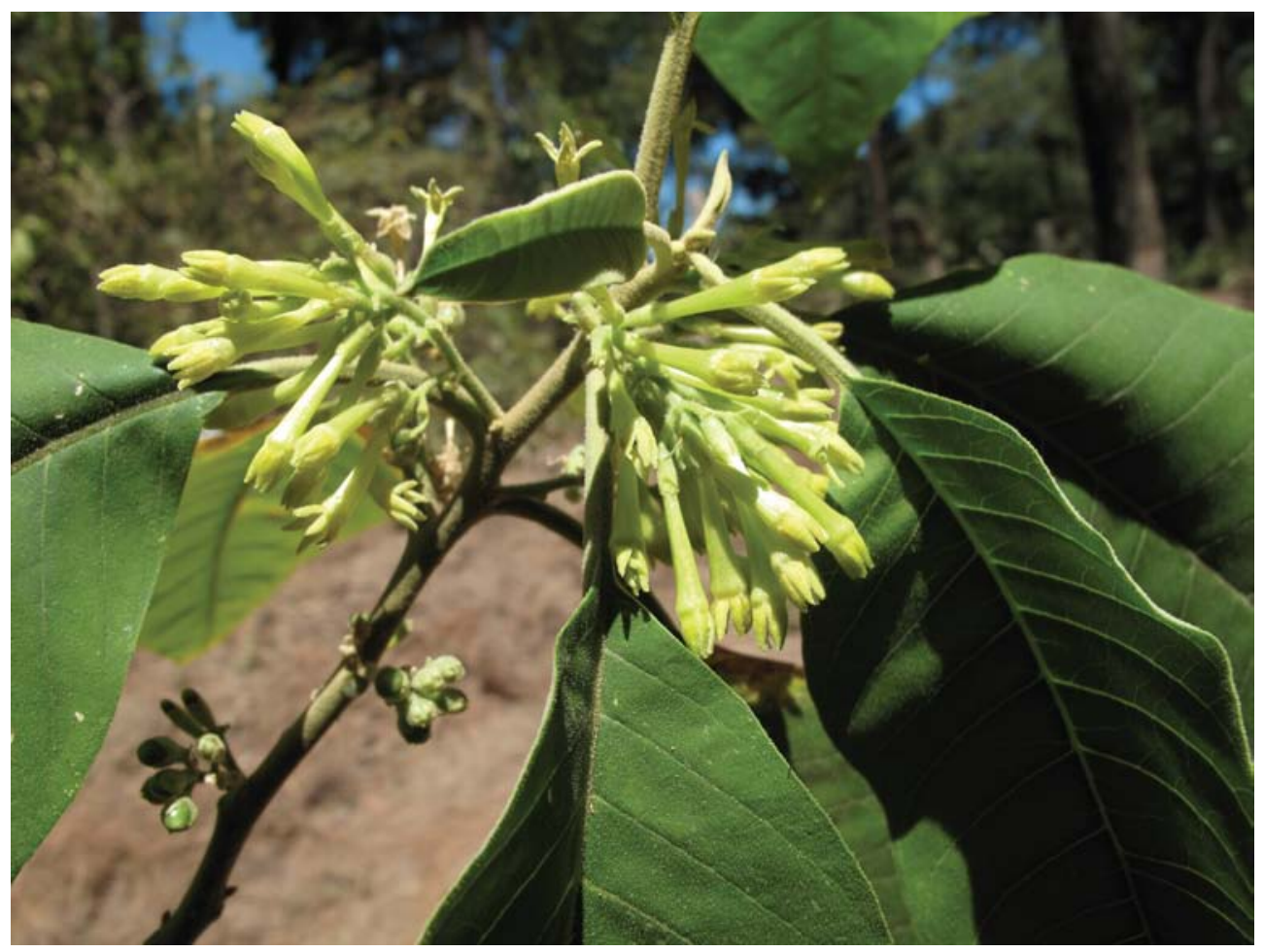

Fig. 2. Cestrum mortonianum var. jardelii. Acercamiento de flores y hojas. 
5, ovados a anchamente ovados, 3.5-5 mm de largo con los márgenes revolutos y traslapándose, el tubo 10-18 mm de largo, 0.5-0.8 mm de ancho en su base y 3-4 mm en su ápice; estambres 5, llegando a la altura de la garganta; filamentos en 3/4 de su longitud adnados al tubo y pilósulos, la parte libre de los filamentos recta, glabra y de 4-5 mm de largo, en el punto entre la parte adnada y parte libre con un apéndice pilósulo de 0.5-0.7 mm de largo; anteras ovadas, dorsifijas, 1-1.6 mm de largo, equinuladas; ovario subgloboso a globoso, 1.2-1.7 mm de diámetro, amarillo, con placentación axilar; óvulos de 5-16; estilo de 13-16 mm de largo; estigma subcupuliforme a subgloboso, 0.7-1 mm de diámetro; infrutescencias, 3-6 cm de largo; frutos elipsoides a globosos, 7-8 mm de largo, morados un poco antes de madurar y negros cuando maduros, glabros; semillas 5-14 por fruto, 2.8-4.5 mm de largo, 1.5-2.5 mm de ancho, castañas oscuras a negras, con la superficie rugosa y reticulada.

Tipo: México, Jalisco: cañada del Tecolote, 1-1.5 km al SO de Corralitos, Mpio. Autlán de Navarro, bosque mesófilo de montaña, 19³6'40.4" N, 104¹8'23.7" O, 1850 m s.n.m., 05/04/2007, Cuevas y Núñez 8902 (holotipo: ZEA).

Paratipos: México, Jalisco: 1-2 km al SO de Corralitos, Mpio. Autlán de Navarro, bosque mesófilo de montaña, 1600-1850 m s.n.m., 13/07/1988, Cuevas y Núñez 3027 (ZEA); 09/03/1991, Guzmán, Sánchez y Cuevas 1260 (ZEA); 27/02/1996, Cuevas, Guzmán y Sánchez 5079 (ZEA); 19/06/1996, Cuevas y Sánchez 5158 (WIS, ZEA); 24/04/2010, Cuevas 9900 (ZEA).

Hábitat: se le encuentra en altitudes de 1600-1900 m, en bosque mesófilo de montaña, junto con Conostegia volcanalis Standl. \& Steyerm., Quercus xalapensis Humb. \& Bonpl., Cornus disciflora Sessé \& Moc. ex DC., Persea hintonii C.K. Allen, Tilia americana L. var. mexicana (Schltdl.) Hardin, Nectandra rudis C.K. Allen, Juglans major (Torr.) A. Heller var. glabrata Manning, Clusia salvinii Donn. Sm., Calatola laevigata Standl., Symplococarpon purpusii (Brandegee) Kobuski, Synardisia venosa (Mast.) Lundell, Prunus cortapico Kerber ex Koehne, Ostrya virginiana (Mill.) K. Koch, Leandra subseriata (Naudin) Cogn., Cedrela odorata L., Arachnothryx manantlanensis (Lorence) Borhidi y Fraxinus uhdei (Wenz.) Lingelsh., entre otras.

Florece de febrero a abril y fructifica de junio a julio.

C. mortonianum var. jardelii es nombrada en honor de Enrique Jardel Peláez, uno de los investigadores de la Universidad de Guadalajara cuyo trabajo hizo posible 
que la Sierra de Manantlán fuera decretada Reserva de la Biosfera, área natural protegida en la cual se mantienen poblaciones de la variedad que se describe. Sus investigaciones han sido importantes para entender el efecto de perturbaciones como el aprovechamiento forestal y el fuego sobre la estructura y diversidad de comunidades vegetales.

Durante el proceso de revisión se encontró material recolectado en el estado de Guerrero y depositado en IEB y MEXU, que pertenece a la variedad típica, y que a diferencia del taxon que se propone como nuevo, conserva las particularidades de las poblaciones de Guatemala, como es el indumento dendroide en el exterior del tubo de la corola. Previo a este registro, C. mortonianum var. mortonianum sólo se había recolectado en el macizo montañoso de Guatemala-Chiapas, y con su hallazgo en el estado de Guerrero se amplía su distribución conocida hasta la Sierra Madre del Sur.

Clave para separar las especies de Cestrum con pubescencia ramificada de México.

1 Cáliz de la mitad del tamaño de la corola o un poco más, de 7-8 mm de largo; Isla Socorro C. pacificum

1 Cáliz de menos de la mitad del tamaño de la corola, de 3.5-6 mm de largo; zona continental 2

2 Filamentos geniculados, de 3-3.5 mm de largo; lóbulos de la corola de $2.5 \mathrm{~mm}$ de largo C. tomentosum

2 Filamentos rectos, de 4-5 mm de largo; lóbulos de la corola de 3.5-5 mm de largo 3

3 Tubo de la corola externamente dendroide-tomentoso C. mortonianum var. mortonianum

3 Tubo de la corola externamente glabro C. mortonianum var. jardelii

\section{MATERIAL ADICIONAL EXAMINADO}

Cestrum mortonianum var. mortonianum. México, Guerrero: $12 \mathrm{~km}$ al E de Guayameo, Mpio. Zirándaro, 18¹8'10.29" N, 101²'19.81" O, 1260 m s.n.m., 21/03/1983, W. E. Martínez S. y J. C. Soto 3626 (IEB, MEXU); Malinaltepec, Mpio. Malinaltepec, $17^{\circ} 09^{\prime} 38.94 "$ N, 101²4'5.51" O, 1900 m s.n.m., 8/02/1991, I. Wagen- 
breth 531 (MEXU). Chiapas: Rancho Nuevo, 6 km al SE de El Rosario, Mpio. Motozintla, 2800 m s.n.m., 2/02/1987, E. Ventura y E. López 4260 (IEB).

\section{AGRADECIMIENTOS}

Al M. C. Enrique V. Sánchez Rodríguez, por la elaboración de la ilustración, a Clotilde Rodríguez Guerrero por la edición de la misma y al M. C. Carlos Palomera García por la elaboración del abstract. El Consejo Nacional de Ciencia y Tecnología y el Consejo Estatal de Ciencia y Tecnología del Estado de Jalisco, apoyaron el proyecto "Estructura, diversidad y reservorios de carbono de la vegetación de cañadas en el Pacífico Mexicano e Inventarios florísticos y estudios estructurales y de diversidad de la vegetación de la costa sur de Jalisco y el estado de Colima”.

\section{LITERATURA CITADA}

D’Arcy, W. G. 2001. Solanaceae. In: Stevens, W. D., C. Ulloa U., A. Pool y O. M. Montiel (eds.). Flora de Nicaragua. Missouri Botanical Garden Press. St. Louis, Missouri, E.U.A. pp. 2376-2426.

Francey, P. 1935. Monographie du genre Cestrum L. Candollea 6: 46-398.

Gentry, Jr., J. L. y P. C. Standley. 1974. Solanaceae: In: Gentry, Jr., J. L. y P. C. Standley (eds.). Flora of Guatemala - Part X, Numbers 1 y 2. Fieldiana Bot. 24(10/1-2): 1-151.

Nee, M. 1986. Solanaceae I. Flora de Veracruz 49: 191 pp.

Nee, M. 2001. An overview of Cestrum. In: van den Berg, R. G., G. W. M. Barendse, G. M. van den Weerden y C. Marinni (eds.). Solanaceae V: Advances in taxonomy and utilization. Nijmegen University Press. Nijmegen. pp. 109-136.

Nee, M. en prep. Nomenclatural synopsis of genus Cestrum. Distribuido por el autor. Nueva York. 73 pp.

Vázquez, J. A., R. Cuevas, T. S. Cochrane, H. H. Iltis, F. J. Santana y L. Guzmán. 1995. Flora de Manantlán. Sida Bot. Miscelany 13: 312 pp. 